\title{
Biological and Cognitive Frameworks for a Mental Timeline
}

\author{
Catalin V. Buhusi ${ }^{1 *}$, Sorinel A. Oprisan ${ }^{2}$ and Mona Buhusi ${ }^{1}$ \\ ${ }^{1}$ Interdisciplinary Program in Neuroscience, Department of Psychology, USTAR Biolnnovations Center, Utah State University, \\ Logan, UT, United States, ${ }^{2}$ Department of Physics and Astronomy, College of Charleston, Charleston, SC, United States
}

Keywords: time perception, temporal order, computer simulations, biologically inspired cognitive architectures, brain, neural networks

\begin{abstract}
Animals are molded by natural forces they do not comprehend. To their minds there is no past and no future... only the everlasting present of a single generation, its trails in the forest, its hidden pathways in the air and in the sea... There is nothing in the Universe more alone than Man. He has entered into the strange world of history...
\end{abstract}

- Loren Eiseley (1960)

\section{INTRODUCTION}

Historians like to order long-gone events in time. When events correlate with years-numbers-events seem to follow a clear time line, but when their order is unclear, historians order events using extra information from folklore, writings, artifacts, and cultural habits. Here we ask the following question: How does the brain, at a neuromechanistic level, order events on a mental time line? This question is relevant to many neuroscience paradigms such as rate calculation, planning, and decision making, processes that crucially depend on the order of events. For example, episodic memory incorporates order and duration of the events in the episode (Tulving and Donaldson, 1972; Eichenbaum, 2017). Events and their features (order, duration, content etc.) are stored in memory and recalled when needed. But how is the order of events assessed when events are recalled from memory to be placed on the timeline? To address this question, we discuss several classes of models of timing and time perception, and their capability of ordering events in time. Because the mental time includes all durations, our discussion will freely mix time scales: milliseconds, seconds, hours, days. Moreover, here we do not discuss in depth the scalar property - the increase in timing errors with the criterion time-because almost all models of timing can reproduce the scalar property, making it a weak criterion for selecting among these models.

\section{COGNITIVE FRAMEWORKS}

Cognitive models of time perception readily implement the "mental timeline" paradigm even when they use an internal representation of time which is very much not timeline-like.

\section{Pacemaker-Accumulator Models}

The Internal Clock Model (Treisman, 1963) and Scalar Expectancy Theory (Gibbon, 1977) assume that time is represented subjectively by the number of accumulated pacemaker pulses (black line in Figure 1A). An alternative monotonic function proposed to link the objective and subjective representations of time is the logarithmic function (red curve in Figure 1A) (for a discussion see 
Staddon and Higa, 1999). Because the subjective time is a monotonically increasing function of the objective time, and because events are stored in memory as numbers which carry intrinsic ordinal information, in these models the order of events is naturally preserved in memory. Moreover, in the Scalar Expectancy Theory subjects can also manipulate these numbers, such that the duration of the interval between two events is readily available as $t_{2}-t_{1}$ (Church, 1984; Gibbon and Church, 1990). Therefore these models readily embody the "mental timeline" paradigm (Church, 1978).

\section{Pacemaker-Free Models}

To tell time, historians use radiocarbon-dating, a method in which the age of an object is estimated based on $\mathrm{C}^{14}$ radioactive decay. Interestingly, the brain's circadian system uses a similar system to tell the time of day based on protein degradation (Golombek and Rosenstein, 2010). Such timing mechanisms motivated a distinct set of cognitive models which rely on monotonically decaying functions, such as the Adaptive Decay Model (Dragoi et al., 2003) (the blue curve in Figure 1A) and the Multiple Time Scales Model (Staddon and Higa, 1999) (Figure 1B). Because time is coded by (one or many) monotonically decaying functions, these models can order events in time simply by comparing the numbers/patterns corresponding to the events.

\section{Distributed Models}

While Pacemaker-Accumulator Models represent time by storing only one piece of information (the number of pulses), real physical systems, e.g., mechanical wristwatches, keep track of time using multiple mechanisms working at different time scales, e.g., an hour-hand, a minute-hand, and a seconds-hand. This motivated the development of cognitive models of timing using multiple non-monotonic distributed processes which evolve at different speeds (scales), such as the Spectral Timing Model (Grossberg and Schmajuk, 1989; Buhusi and Schmajuk, 1999; Figure 1C). In this model events are represented by distinct nonmonotonic patterns of memory traces. Because traces evolve at different speeds, they can be correctly ordered on a time line, $t_{1}<t_{2}$, in a manner similar to comparing the pattern of the hands on the wristwatch with a desired time, despite using an internal representation of time which is very much not timeline-like.

\section{BIOLOGICAL FRAMEWORKS}

While cognitive models readily order events on a time line, biologically-inspired models have difficulties ordering events

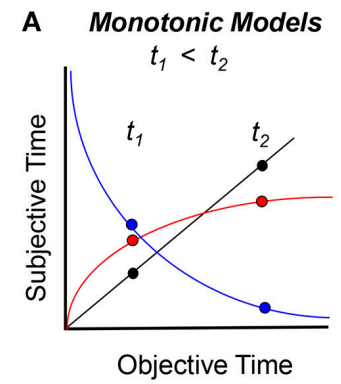

D

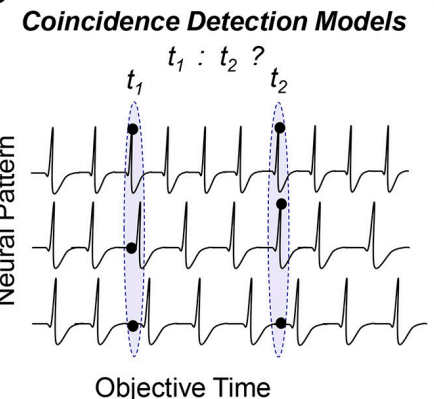

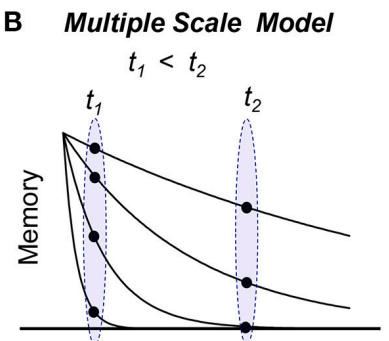

Objective Time

E

State Dependent Models $t_{1}: t_{2}$ ?

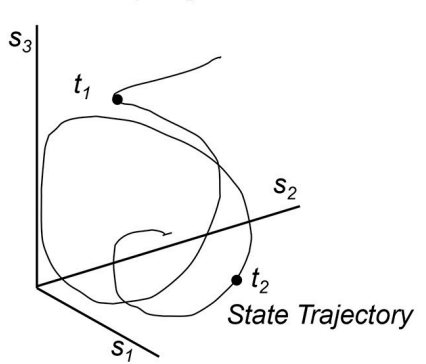

C Spectral Timing Model

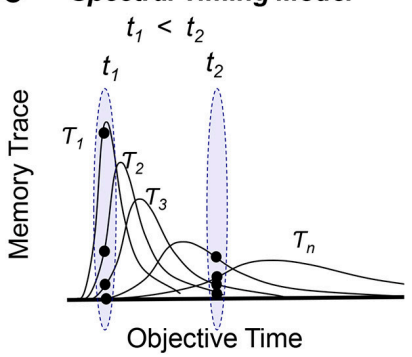

F

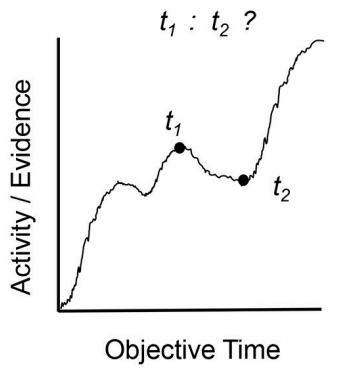

FIGURE 1 | Temporal order within the framework of cognitive (A-C) and biological (D-F) models of timing and time perception. Panels indicate how models assess temporal order of two events at times $t_{1}$ and $t_{2}$ (see text for details) (A) Subjective time is a monotonic function of objective time (linear-black, logarithmic-red, or exponentially decaying - blue), such that the objective order of events can be inferred from the subjective representation of time, $t_{1}<t_{2}$. (B) Multiple monotonic (exponentially-decaying) memory traces can convey temporal order. (C) Multiple non-monotonic traces that evolve at different speeds can also convey temporal order. (D) An internal representation of time based on patterns of firing neurons cannot in itself convey temporal order, as patterns have no intrinsic order. (E) Rather than being a coordinate, time could be considered a parameter of a system that follows a trajectory in a state-based coordinate system $\left\{s_{1}, s_{2}, \ldots, s_{n}\right\}$. Such systems can equally follow the same state trajectory toward the future or toward the past, thus, they have difficulty ordering events in time. (F) The pattern of activity of a population of neurons varies in time as the model accumulates evidence; evidence / activity / patterns correlate with time but are not solely representing time. Biological models (D-F) need extra assumptions / transformations / information to map activity / states / evidence / patterns to order of events; such information may be provided by chemical, electrical, and circuit level constraints rather than time itself. 
because they process and store events in memory as neural patterns, which lack intrinsic ordinal value.

\section{Coincidence Detection Models}

A class of biologically-inspired timing models assume that time is represented by the coincidental activation of multiple neuronal inputs. For example, in the Coincidence Detection Model (Miall, 1989) and in the Striatal Beat Frequency Model (Matell and Meck, 2004; Buhusi and Meck, 2005), timing is coded by the pattern of multiple neuronal oscillators (Figure 1D). Supplemental assumptions are used to map the models onto the brain: For example, the Striatal Beat Frequency Model ascribes a role for detecting event durations to medium spiny neurons within the dorsal striatum which become entrained to fire in response to oscillating, coincident cortical inputs. Interestingly, no extra assumptions are needed to describe the scalar property, which emerges in the Striatal Beat Frequency Model due to neural noise (Buhusi and Oprisan, 2013; Oprisan and Buhusi, 2013a,b, 2014). However, when events are recalled, these models have difficulty assessing the order of events, as there is no predetermined order of neural patterns. These models need extra information to order of events in time, which may be provided by circuit level constraints, such as the unidirectionality of action potentials.

\section{State Dependent Models}

Another way real physical systems code for time is in their (distributed) state. For example, winter is different from summer in all the changes in foliage, temperature, precipitation etc. Similarly, in the State Dependent Timing Model (Buonomano and Maass, 2009) the system follows a trajectory along which states (events) are coded in time (Figure 1E). When events (states) are recalled from memory, the model has difficulty ordering events, pretty much like one has difficulty saying whether summer follows winter or rather winter follows summer. In fact, state dependent models can follow the same trajectory "forward" in time, as well as "backward" in time, since time is a parameter rather than a coordinate in these models. Thus, state dependent models are physically- and biologically-inspired, but need extra information to implement a unidirectional timeline. Extra information to order events in time may be provided by chemical reactions, as not all chemical reactions are bidirectional; this type of information may limit the trajectory of the system, and provide a sense of order.

\section{Evidence Accumulation Models}

Another set of biologically-inspired timing models take advantage of the observation that during an interval the activity of specific populations of neurons largely increases as shown in Figure 1F (Leon and Shadlen, 2003; Mita et al., 2009; Xu et al., 2014). This (non-linear) increase in activity was suggested to reflect neuronal integration (Simen et al., 2011), drift-diffusion processes (Luzardo et al., 2013), or accumulation of evidence (Leon and Shadlen, 2003). Notably, the similarity between Evidence Accumulation Models (Figure 1E) and Pacemaker-Accumulator Models (Figure 1A) is misleading. The latter assume that time is stored in memory as (ordered) numbers, while the former store in memory the patterns of neural activation/evidence, supposedly devoid of order. Not only Evidence Accumulation Models work with patterns, but the nature of the information manipulated/stored (activation or evidence) is different than in Pacemaker-Accumulator Models (pulses or numbers). Evidence Accumulation Models can compare events in terms of evidence/patterns of activation, but not necessarily in time. It would require an extra assumption (transformation) to map activation or evidence into order of events. For example one could assume that more activity/evidence represents a later event, but whether the brain follows this assumption or not it is not known at this time.

The brain seems to need extra sources of information-at the chemical, electrical, circuit level-than time itself to order memory patterns in a time line. This idea is consistent with recent experimental evidence suggesting that time and order of events are coded by different processes in the brain (D'argembeau et al., 2015).

\section{CONCLUSIONS}

While cognitive models of timing and time perception seem readily equipped to represent order of events on a mental time line, they do not do so in a realistic manner. Meantime, biologically-plausible models face specific challenges when ordering events into "past," "present" and "future." However, as an electro-chemical network, the brain may impose its own chemical, electrical, and circuit level constraints that could provide a sense of order other than time itself. Either way, it seems that more work needs to be done at both ends of the spectrum of theories, toward an explanation of the mental time line that is biologically realistic. One major objective of a science of the mental time should be to incorporate orderand not necessarily number-into biologically-inspired theories of timekeeping to understand how the brain represents time and order on the mental timeline. We speculate that the apparent mental time line readily embodied by Scalar Expectancy Theory is represented in the brain in a distributed manner-much as in the Spectral Timing Model (Grossberg and Schmajuk, 1989; Buhusi and Schmajuk, 1999)-at multiple brain regions, including striatum (Bakhurin et al., 2017), amygdala (Dallérac et al., 2017), hippocampus (Eichenbaum, 2014), lateral intra-parietal sulcus (Jazayeri and Shadlen, 2015), insula (Wittmann et al., 2010), and even visual areas (Shuler, 2016). In turn, these brain regions may use their own system to code the passage of time, possibly similar to Coincidence Detection Models (Matell and Meck, 2004; Buhusi and Meck, 2005), State Dependent Models (Buonomano and Maass, 2009), or Evidence Accumulation Models (Leon and Shadlen, 2003; Simen et al., 2011; Luzardo et al., 2013) discussed above. In this scenario, timing is a process distributed at multiple levels, molecular, local circuits, and brain-wide circuits (Buhusi et al., 2016) that generates a linear timeline at the behavioral level from multiple non-linear local timelines. Future research should differentiate and integrate a "sense of time passage" with "a sense of order" of events and their biological substrates that enable the (re)construction of a mental time line. 


\section{AUTHOR CONTRIBUTIONS}

All authors listed have made a substantial, direct and intellectual contribution to the work, and approved it for publication.

\section{REFERENCES}

Bakhurin, K. I., Goudar, V., Shobe, J. L., Claar, L. D., Buonomano, D. V., and Masmanidis, S. C. (2017). Differential encoding of time by prefrontal and striatal network dynamics. J. Neurosci. 37, 854-870. doi: 10.1523/JNEUROSCI.1789-16.2016

Buhusi, C. V., and Meck, W. H. (2005). What makes us tick? Functional and neural mechanisms of interval timing. Nat. Rev. Neurosci. 6, 755-765. doi: $10.1038 / \mathrm{nrn} 1764$

Buhusi, C. V., and Oprisan, S. A. (2013). Time-scale invariance as an emergent property in a perceptron with realistic, noisy neurons. Behav. Processes 95, 60-70. doi: 10.1016/j.beproc.2013.02.015

Buhusi, C. V., Oprisan, S. A., and Buhusi, M. (2016). Clocks within clocks: timing by coincidence detection. Curr. Opin. Behav. Sci. 8, 207-213. doi: 10.1016/j.cobeha.2016.02.024

Buhusi, C. V., and Schmajuk, N. A. (1999). Timing in simple conditioning and occasion setting: a neural network approach. Behav. Processes 45, 33-57. doi: 10.1016/S0376-6357(99)00008-X

Buonomano, D. V., and Maass, W. (2009). State-dependent computations: spatiotemporal processing in cortical networks. Nat. Rev. Neurosci. 10, 113-125. doi: $10.1038 / \mathrm{nrn} 2558$

Church, R. M. (1978). "The internal clock," in Cognitive Processes in Animal Behavior, eds S. H. Hulse, H. Fowler, and W. K. Honig (Hillsdale, NJ: Erlbaum), 277-310.

Church, R. M. (1984). Properties of an internal clock. Ann. N.Y. Acad. Sci. 423, 566-582. doi: 10.1111/j.1749-6632.1984.tb23459.x

Dallérac, G., Graupner, M., Knippenberg, J., Martinez, R. C., Tavares, T. F., Tallot, L., et al. (2017). Updating temporal expectancy of an aversive event engages striatal plasticity under amygdala control. Nat. Commun. 8:13920. doi: $10.1038 /$ ncomms 13920

D’argembeau, A., Jeunehomme, O., Majerus, S., Bastin, C., and Salmon, E. (2015). The neural basis of temporal order processing in past and future thought. $J$. Cogn. Neurosci. 27, 185-197. doi: 10.1162/jocn_a_00680

Dragoi, V., Staddon, J. E., Palmer, R. G., and Buhusi, C. V. (2003). Interval timing as an emergent learning property. Psychol. Rev. 110, 126-144. doi: 10.1037/0033-295X.110.1.126

Eichenbaum, H. (2014). Time cells in the hippocampus: a new dimension for mapping memories. Nat. Rev. Neurosci. 15, 732-744. doi: 10.1038/nrn3827

Eichenbaum, H. (2017). On the integration of space, time, and memory. Neuron 95, 1007-1018. doi: 10.1016/j.neuron.2017.06.036

Eiseley, L. (1960). The long loneliness: man and the porpoise: two solitary destinies. Am. Scholar 30, 57-64.

Gibbon, J. (1977). Scalar expectancy theory and Weber's law in animal timing. Psychol. Rev. 84, 279-325. doi: 10.1037/0033-295X.84.3.279

Gibbon, J., and Church, R. M. (1990). Representation of time. Cognition 37, 23-54. doi: 10.1016/0010-0277(90)90017-E

Golombek, D. A., and Rosenstein, R. E. (2010). Physiology of circadian entrainment. Physiol. Rev. 90, 1063-1102. doi: 10.1152/physrev.0000 9.2009

Grossberg, S., and Schmajuk, N. A. (1989). Neural dynamics of adaptive timing and temporal discrimination during associative learning. Neural Netw. 2, 79-102. doi: 10.1016/0893-6080(89)90026-9

Jazayeri, M., and Shadlen, M. N. (2015). A neural mechanism for sensing and reproducing a time interval. Curr. Biol. 25, 2599-2609. doi: 10.1016/j.cub.2015.08.038

\section{FUNDING}

The writing of this article was supported by NIH grants MH073057 to CB, AG038767 and NS090283 to MB, and NSFIOS CAREER award 1054914 to SO.
Leon, M. I., and Shadlen, M. N. (2003). Representation of time by neurons in the posterior parietal cortex of the macaque. Neuron 38, 317-327. doi: 10.1016/S0896-6273(03)00185-5

Luzardo, A., Ludvig, E. A., and Rivest, F. (2013). An adaptive driftdiffusion model of interval timing dynamics. Behav. Processes 95, 90-99. doi: 10.1016/j.beproc.2013.02.003

Matell, M. S., and Meck, W. H. (2004). Cortico-striatal circuits and interval timing: coincidence detection of oscillatory processes. Cogn. Brain Res. 21, 139-170. doi: 10.1016/j.cogbrainres.2004.06.012

Miall, R. C. (1989). The storage of time intervals using oscillating neurons. Neural Comput. 1, 359-371. doi: 10.1162/neco.1989.1.3.359

Mita, A., Mushiake, H., Shima, K., Matsuzaka, Y., and Tanji, J. (2009). Interval time coding by neurons in the presupplementary and supplementary motor areas. Nat. Neurosci. 12, 502-507. doi: 10.1038/nn.2272

Oprisan, S. A., and Buhusi, C. V. (2013a). How noise contributes to time-scale invariance of interval timing. Phys. Rev. E Stat. Nonlin. Soft Matter Phys. 87:052717. doi: 10.1103/PhysRevE.87.052717

Oprisan, S. A., and Buhusi, C. V. (2013b). Why noise is useful in functional and neural mechanisms of interval timing? BMC Neurosci. 14:84. doi: 10.1186/1471-2202-14-84

Oprisan, S. A., and Buhusi, C. V. (2014). What is all the noise about in interval timing? Philos. Trans. R. Soc. Lond. B. Biol. Sci. 369:20120459. doi: 10.1098/rstb.2012.0459

Shuler, M. G. (2016). Timing in the visual cortex and its investigation. Curr. Opin. Behav. Sci. 8, 73-77. doi: 10.1016/j.cobeha.2016.02.006

Simen, P., Balci, F., De Souza, L., Cohen, J. D., and Holmes, P. (2011). A model of interval timing by neural integration. J. Neurosci. 31, 9238-9253. doi: 10.1523/JNEUROSCI.3121-10.2011

Staddon, J. E., and Higa, J. J. (1999). Time and memory: towards a pacemaker-free theory of interval timing. J. Exp. Anal. Behav. 71, 215-251. doi: 10.1901/jeab.1999.71-215

Treisman, M. (1963). Temporal discrimination and the indifference interval. Implications for a model of the "internal clock". Psychol. Monogr. 77, 1-31. doi: $10.1037 / \mathrm{h} 0093864$

Tulving, E., and Donaldson, W. (eds.). (1972). Organization of Memory. New York, NY: Academic Press.

Wittmann, M., Simmons, A. N., Aron, J. L., and Paulus, M. P. (2010). Accumulation of neural activity in the posterior insula encodes the passage of time. Neuropsychologia 48, 3110-3120. doi: 10.1016/j.neuropsychologia.2010.06.023

Xu, M., Zhang, S. Y., Dan, Y., and Poo, M. M. (2014). Representation of interval timing by temporally scalable firing patterns in rat prefrontal cortex. Proc. Natl. Acad. Sci. U.S.A. 111, 480-485. doi: 10.1073/pnas.1321314111

Conflict of Interest Statement: The authors declare that the research was conducted in the absence of any commercial or financial relationships that could be construed as a potential conflict of interest.

Copyright (C) 2018 Buhusi, Oprisan and Buhusi. This is an open-access article distributed under the terms of the Creative Commons Attribution License (CC $B Y)$. The use, distribution or reproduction in other forums is permitted, provided the original author(s) and the copyright owner are credited and that the original publication in this journal is cited, in accordance with accepted academic practice. No use, distribution or reproduction is permitted which does not comply with these terms. 